\title{
Métodos de estimativa da evapotranspiração de referência para as condições do semiárido Nordestino
}

\section{Methods of estimation of reference crop evapotranspiration for the conditions of northeastern semiarid, Brazil}

\author{
Edmilson Gomes Cavalcante Junior ${ }^{1 *}$; Alexsandra Duarte Oliveira ${ }^{2}$; Bruno Marçal \\ de Almeida ${ }^{3}$; José Espínola Sobrinho ${ }^{4}$
}

\section{Resumo}

O objetivo deste trabalho foi avaliar métodos de estimativa da evapotranspiração de referência (ETo) em condições de baixa e elevada umidade relativa usando o método de Penman Monteith-FAO 56 como referência. Foram utilizados dados de uma estação meteorológica convencional pertencente à Universidade Federal Rural do Semiárido, em Mossoró-RN referentes ao período de janeiro de 2002 a junho de 2008. A avaliação do desempenho foi realizada por meio do índice de precisão " $r$ ", um índice de exatidão “ $d$ ", e de confiança ou desempenho " $c$ ". Os métodos Kimberly-Penman 1982, Kimberly-Penman 1972, Benevides-Lopez 1970 e Hargreaves-Samani 1985 tiveram um aumento substancial nos seus desempenhos quando utilizados em condições de elevada umidade (UR > 70\%), em comparação com as condições de baixa umidade (UR $<70 \%$ ). As equações de Makkink 1957 e Hamon 1961 subestimaram a ETo em ambos os períodos e o método Penman modificado-FAO24 tendeu a superestimar o método padrão. Os métodos Penman 1948, FAO24-Radiação, FAO24-Blaney-Criddle e Frere-Popov apresentaram melhor desempenho entre os avaliados.

Palavras-chave: Penman Monteith-FAO 56, umidade, Eto

\begin{abstract}
The objective of this work was to evaluate methods of estimation of reference crop evapotranspiration (ETo) in conditions of low and high air relative humidity, using the Penman Monteith-FAO 56 method as reference. Data from the period between January 2002 and June 2008 were obtained from a conventional meteorological station at Semiarid Federal Rural University, in Mossoró, RN, Brazil. Evaluation of methods was made by means of the precision index " $r$ ", accuracy " $d$ ", and of confidence or performance " $c$ ". Methods Kimberly-Penman 1982, Kimberly-Penman 1972, Benevides-Lopez 1970 and Hargreaves-Samani 1985 obtained an important improvement in their performance when high air relative humidity conditions were considered (UR $>70 \%$ ), compared to low air relative humidity conditions (UR < 70\%). Equations of Makkink 1957 and Hamon 1961 underestimated ETo in both periods, and the method Penman modified-FAO24 showed tendency to overestimate the reference method. Methods Penman 1948, FAO24-Radiation, FAO24-Blaney-Criddle and Frere-Popov presented the higher performance among the evaluated methods.
\end{abstract}

Key words: Penman Monteith-FAO 56, humidity, Eto

1 Tecnólogo em Irrigação e Drenagem, Mestrando em Irrigação e Drenagem, UFERSA, BR 110 do km 47, Bairro: Costa e Silva, 59625-900, Mossoró, RN. E-mail: ediguatu@yahoo.com.br

2 Pesquisadora A, Embrapa Cerrados, BR 020, km 18, C P 08223, 73310-970, Planaltina, DF. E-mail: alexsandra.duarte@cpac. embrapa.br

3 Tecnólogo em Irrigação e Drenagem, Mestrando em Irrigação e Drenagem. Universidade Federal Rural do Semi-Árido, UFERSA. BR 110 do km 47, Bairro: Costa e Silva, 59625-900, Mossoró, RN, E-mail: brunoevapo@gmail.com

${ }^{4}$ Eng $^{\mathrm{o}}$ Agro ${ }^{\circ}$, Prof. Dr. DCAT, UFERSA, Mossoró, RN. E-mail: jespinola@ufersa.edu.br

* Autor para correspondência

Recebido para publicação 28/03/2010 Aprovado em 14/09/201 1 


\section{Introdução}

O conhecimento das necessidades hídricas das culturas é de grande importância para o estudo do manejo da água de irrigação. A evapotranspiração é uma das principais variáveis do ciclo hidrológico. A taxa de evapotranspiração refere-se à transferência de água para a atmosfera, da superfície do solo e das plantas pela combinação da evaporação com a transpiração (SEDIYAMA, 1998).

A estimativa do consumo hídrico das culturas tem sido tema de vários estudos nas últimas décadas, várias foram às metodologias criadas para tentar representar de maneira precisa essa questão. A obtenção deste consumo pode ser realizada através de medições diretas no campo ou por meio indireto através de equações empíricas. As medições diretas muitas vezes requerem a utilização de equipamentos sofisticados e caros o que no geral inviabiliza sua utilização. Por isso, tem-se recorrido à utilização de equações empíricas, por serem mais práticas e viáveis de serem usadas para fins de manejo da irrigação.

Segundo Allen et al. (1998), vários são os métodos empíricos criados, por vários cientistas e pesquisadores, para determinação da ET utilizando diferentes elementos climáticos. No entanto, tais métodos somente estimam de forma satisfatória a evapotranspiração nas condições de clima em que são desenvolvidos, e quando utilizados em condições diferentes podem proporcionar grandes erros e gerar grandes perdas nas produções ou desperdício de recursos hídricos. Com isso, a FAO observou a necessidade de se obter um método padrão que se adaptasse a diferentes localidades e climas, criando o método Penman-Monteith FAO 56 (PM FAO-56) que foi derivado da equação original de Penman (1948).

O método PM FAO-56 é bastante utilizado em trabalhos para avaliar e calibrar diferentes equações empíricas como em Oliveira et al. (2005), Oliveira e Carvalho (1998) e Vescove e Turco (2005). Embora seja um método complexo, concilia os aspectos aerodinâmicos e termodinâmicos, incluindo a resistência ao transporte de calor sensível e de vapor d'água e a resistência da superfície à transferência de vapor d'água, fatores que nenhum outro método leva em consideração e que proporciona melhor precisão (OLIVEIRA, 2003). De acordo com Medeiros (2002), diversos trabalhos científicos têm mostrado que o desempenho do método de PM FAO-56 na estimativa da ETo é satisfatório, quando comparado com medidas lisimétricas. No entanto, muitas vezes nem todos os elementos meteorológicos necessários para o uso desse modelo encontram-se disponíveis. Nessa situação, outros métodos que necessitem de um número menor de elementos meteorológicos podem ser usados (FIETZ; SILVA; URCHEI, 2005).

A proposta deste trabalho foi avaliar o comportamento de diferentes equações empíricas na estimativa da ETo, em condições de meses úmidos e secos, no município de Mossoró-RN, tendo como padrão o método Penman-Monteith FAO 56.

\section{Material e Métodos}

Os dados utilizados neste estudo foram coletados pelaestação meteorológica convencional pertencente à Universidade Federal Rural do Semiárido, em Mossoró-RN, cujas coordenadas geográficas são: latitude $5^{\circ} 12^{\prime} 48^{\prime \prime} \mathrm{S}$, longitude $37^{\circ} 18^{\prime} 44^{\prime}$ ' W. Grw. e altitude de $37 \mathrm{~m}$. Segundo W. KÖEPPEN, citado por Carmo Filho et al. (1991), a classificação climática local, é do tipo Bswh', ou seja, clima seco e quente com as maiores precipitações no verão atrasando-se para o outono. Pela classificação de W. C. THORNTHWAITE o clima de Mossoró é do tipo DdA'a', ou seja semi-árido, megatérmico com pequeno ou nenhum excesso de água durante o ano (IZÍDIO, 1994).

Foram utilizados dados diários de temperatura máxima e mínima, umidade relativa, velocidade do vento, evaporação do tanque classe A e insolação, compreendidos de janeiro de 2002 a junho de 2008. Por meio do programa REF-ET versão 2.01.17, desenvolvido por Allen (2001) foram 
feitas as estimativas da ETo dos seguintes métodos: Kimberly-Penman segundo Wright (1982), Kimberly-Penman segundo Wright e Jensen (1972), Penman (1948), Penman modificado-FAO24, Frere \& Popov (1979), FAO24-Radiação, FAO24-BlaneyCriddle, FAO24-Tanque Classe A, HargreavesSamani (1985), Priestley-Taylor (1972), Makkink (1957), Benevides-Lopez (1970), Jensen-Haise (1963), Camargo (1971) e Hamon (1961). Sendo que os últimos quatro métodos foram estimados diretamente e não pelo REF-ET, por não estarem disponíveis no referido software, sendo calculados pelas seguintes equações:

1) Benevides-Lopez (1970) citado por Medeiros (1998)

'To $=1,21 \cdot 10^{\left(\frac{7,45, T}{243,7+T}\right)} \cdot(1-0,01 \cdot U R)+0,21 \cdot T-2,30 \quad$ Eq. 1

em que: ETo: evapotranspiração de referência $\left(\mathrm{mm} . \mathrm{dia}^{-1}\right)$; T: temperatura média do ar em ${ }^{\circ} \mathrm{C}$; e UR: umidade relativa do ar em $\%$.

2) Jensen-Haise (1963)

\section{$E T o=R s(0,0252 T * 0,078) \quad$ Eq. 2}

em que: T: temperatura média do ar em ${ }^{\circ} \mathrm{C}$; e Rs: radiação solar global à superfície em cal.cm ${ }^{-2} \cdot \mathrm{dia}^{-1}$.

3) Camargo (1971)

\section{ETo $=0,01 R a T K \quad$ Eq. 3}

em que: T: temperatura média do ar em ${ }^{\circ} \mathrm{C}$; Ra: radiação solar extraterrestre $\left(\mathrm{mm} \cdot \mathrm{dia}^{-1}\right)$; e $\mathrm{K}$ : fator de ajuste de Camargo.

4) Hamon (1961)

ETo $=0,55\left(\frac{N}{12}\right)^{2}\left(\frac{4,95 \exp ^{0,062 T}}{100}\right) 25,4$

Eq. 4

em que: T: temperatura média do ar em ${ }^{\circ} \mathrm{C}$; e $\mathrm{N}$ : fotoperíodo em $h$.

O método Penman-Montheith FAO 56 foi considerado como padrão, para análise dos demais métodos (Equação 5). A comparação dos métodos foi realizada em dois períodos distintos, período úmido e seco. Foi considerado como período úmido os meses que apresentaram umidade relativa média maior que $70 \%$ e como período seco, os que apresentaram umidade relativa do ar abaixo de $70 \%$.

$$
\text { ETo }=\frac{0,408 \Delta\left(R_{n}-G\right)+\gamma \frac{900}{T_{a}+273} u_{2}\left(e_{s}-e_{a}\right)}{\Delta+\gamma\left(1+0,34 u_{2}\right)}
$$

$\Delta$ : declividade da curva de pressão de vapor no ponto de temperatura média $\left(\mathrm{kPa}^{\circ} \mathrm{C}^{-1}\right)$;

$\mathrm{Rn}$ : radiação solar líquida total do gramado (MJ $\left.\mathrm{m}^{-2} \mathrm{~d}^{-1}\right)$;

$\mathrm{G}$ : fluxo de calor no solo $\left(\mathrm{MJ} \mathrm{m}^{-2} \mathrm{~d}^{-1}\right)$;

$\gamma$ : constante psicrométrica $\left(\mathrm{kPa}^{\circ} \mathrm{C}^{-1}\right)$;

$\mathrm{e}_{\mathrm{s}}$ : pressão de saturação do vapor médio diário $(\mathrm{kPa})$;

$\mathrm{e}_{\mathrm{a}}$ : Pressão atual de vapor médio diário $(\mathrm{kPa})$;

$\mathrm{u}_{2}$ : velocidade do vento média diária a $2 \mathrm{~m}$ de altura $\left(\mathrm{m} \mathrm{s}^{-1}\right)$; e

$\mathrm{T}_{\mathrm{a}}$ : temperatura média do ar $\left({ }^{\circ} \mathrm{C}\right)$.

A análise comparativa entre o método padrão e os avaliados, foi baseada em regressão linear, onde a variável depende foi considerada a ETo do método padrão e a independente a ETo do métodos avaliados, e os seguintes índices estatísticos de comparação sugeridos Camargo e Sentelhas (1997): o grau de precisão foi obtido por meio do coeficiente de correlação “ $r$ " (Equação 6), a exatidão foi avaliada pelo índice de Willmott " $d$ " (Equação 7) e o desempenho pelo indicador " $c$ ". Sendo c obtido pelo produto de $d * r$. Onde os valores de c são classificados conforme a Tabela 1 .

$$
\begin{aligned}
& r=\frac{\sum_{i=1}^{N}(O i-O) \cdot(P i-P)}{\sqrt{\sum_{i=1}^{N}(O i-O)^{2}} \cdot \sqrt{\sum_{i=1}^{N}(P i-P)^{2}}} \\
& d=1-\left[\frac{\sum_{i=1}^{N}(P i-O i)^{2}}{\sum_{i=1}^{N}(|P i-O|+|O i-O|)^{2}}\right]
\end{aligned}
$$


sendo: $P i$ o valor estimado; $\mathrm{P}$ a média do valor estimado; $O i$ o valor observado e $O$ é a média dos valores observados.

A verificação dos erros proporcionados pelas estimativas foi realizada pelo cálculo do erro padrão da estimativa (EPE) conforme Jensen, Burman e Allen (1990).

$$
E P E=\left[\frac{\Sigma\left(Y-Y_{m}\right)^{2}}{n-1}\right]^{0,:}
$$

sendo: $\mathrm{Y}$ a evapotranspiração estimada pelo método avaliado (mm.dia $\left.{ }^{-1}\right)$; $\mathrm{m}$ a evapotranspiração estimada pelo método PM FAO $56\left(\mathrm{~mm} \cdot \mathrm{dia}^{-1}\right)$; e $n$ o número total de observações.

Tabela 1. Valores dos coeficientes de desempenho conforme Camargo e Sentelhas (1997).

\begin{tabular}{ll}
\hline Valor de "c" & Desempenho \\
\hline$>0,85$ Ótimo & \\
0,76 a 0,85 & Muito Bom \\
0,66 a 0,75 & Bom \\
0,61 a 0,65 & Mediano \\
0,51 a 0,60 & Sofrível \\
0,41 a 0,50 & Mau \\
$\leq 0,40$ & Péssimo \\
\hline
\end{tabular}

\section{Resultados e Discussão}

A partir da estimativa da ETo pelos métodos descritos, procurou-se identificar uma equação que melhor se ajustasse ao método de PenmanMonteith FAO 56 e que envolvesse uma quantidade menor de parâmetros nos cálculos, de modo a se obter de forma mais simples a ETo em dois períodos distintos (seco e úmido) em Mossoró-RN. Na maioria dos anos estudados, o período seco (umidade relativa média mensal $<70 \%$ ) esteve compreendido entre os meses de julho a dezembro e o período úmido (umidade relativa média mensal $>70 \%$ ) de janeiro a junho. A média anual da umidade relativa do ar e da ETo estão expostas na Figura 1.

Analisando-se os resultados de acordo com as Tabelas 2 e 3, os métodos Hamon (1961) e Makkink (1957) subestimaram a evapotranspiração nos dois períodos analisados, sendo a subestimativa mais acentuada no período seco. Em relação a Makkink (1957) esse comportamento se deve, provavelmente, ao fato dos coeficientes utilizados da equação original serem desenvolvidos nas condições climáticas de Wageningem, na Holanda e de acordo com Pereira, Villa Nova e Sedyiama (1997), os coeficientes dessa equação podem variar de local para local e nas diferentes escalas de tempo de medida. Vescove e Turco (2005) obtiveram resultado semelhante ao avaliar o método de Makkink (1957) nas condições climáticas de AraraquaraSP. Borges e Mendiondo (2007) avaliaram diferentes metodologias de determinação da ETo na Bacia do Rio Jacupira, SP, e também verificaram que o método de Hamon (1961) subestimou a ETo, em todas as estações do ano.

Observando a Tabela 2, verifica-se que, o EPE para os diferentes modelos avaliados, com base nos valores ETo para o período seco, apresentaram uma variação de 0,19 a 2,74 mm.d $\mathrm{d}^{-1}$, com o menor valor para Penman (1948) e o maior valor, para o método de Hamon (1961). Para o período úmido (Tabela 3) o EPE variou de 0,21 a 0.99 mm.d 1 para os métodos Kimberly-Penman 1972 e FAO24-Tanque Classe A, respectivamente. Esse maior intervalo nos valores do EPE do período seco pode estar associado ao fato de que a maioria dos métodos estudados foram desenvolvidos em condições de demanda evapotranspirativa menores do que na região do presente estudo, tendendo a subestimar os valores de ETo de forma acentuada quando utilizados no período seco, proporcionando assim maiores valores do EPE. Visualizam-se, nas Figuras 2 e 3 a análise 
de regressão linear entre os métodos avaliados e o método padrão, com suas respectivas equações de ajuste e coeficiente de determinação $\left(\mathrm{r}^{2}\right)$. Aos Gráficos foi adicionada uma linha ao meio, a fim de auxiliar a verificação de quando um método sub ou superestima o método padrão. Observase que dentro os métodos avaliados o FAO24Tanque Classe A foi o que apresentou pior correlação em ambos os período analisados.

A equação de Jensen-Haise (1963) segundo Medeiros (2008), foi uma equação desenvolvida para regiões áridas e semi-áridas, o que explica sua boa adaptabilidade para o período seco, onde obteve desempenho ótimo, ao contrário do período úmido quando obteve apenas desempenho mediano. Como descrito por Allen et al. (1998) o método Penman modificadoFAO24 superestima com frequência a ETo em condições de baixa demanda evapotranspirativa, podendo essa atingir níveis de até $20 \%$. Neste trabalho o referido método superestimou em $15,7 \%$ a ETo no período úmido. Apesar dessa elevada superestimativa o método demonstrou ser bastante preciso $(r=0,99)$ obtendo um desempenho muito bom (c $=0,80)$, quando utilizado no período úmido (Tabela 3 ).

Os métodos Kimberly-Penman 1982 e Kimberly-Penman 1972 apresentaram classificações inferiores quando utilizados no período seco em relação ao úmido, o que pode ser observado pelos valores de EPE e pelos coeficientes de desempenho "c", onde Kimberly-Penman 1982 foi classificado como sofrível no período seco e muito bom no úmido, comportamento semelhante aos métodos Benevides-Lopez (1970), Camargo (1971), Priestley-Taylor (1972) e Hargreaves e Samani (1985) que também apresentaram uma melhora significativa quando utilizados no período úmido.

Figura 1. Média anual da ETo, estimada pelo método Penman-Montheith FAO 56, e da umidade relativa do ar, compreendia entre janeiro de 2002 a junho de 2008, para o município de Mossoró, RN.

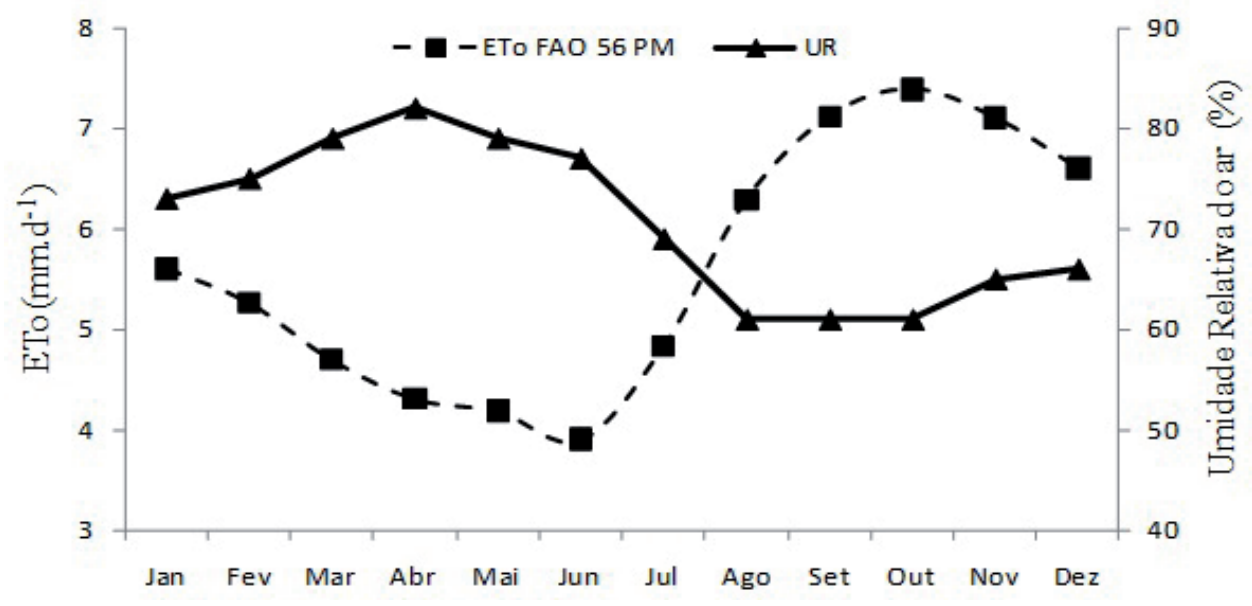


Tabela 2. Valores de coeficiente de correlação, índices de exatidão, coeficientes de desempenho e erro padrão de estimativa, referentes ao período seco em Mossoró-RN, de 2002 a 2008.

\begin{tabular}{lcccccc}
\hline Equações & \% & r & d & c & Desempenho & EPE (mm.dia $\left.\mathbf{m}^{-1}\right)$ \\
\hline Penman 1948 & 102.5 & 0.99 & 0.98 & 0.97 & Ótimo & 0.19 \\
FAO24-Radiação & 102.0 & 0.97 & 0.97 & 0.94 & Ótimo & 0.24 \\
FAO24-Blaney-Cliddle & 97.8 & 0.97 & 0.95 & 0.93 & Ótimo & 0.24 \\
Frere-Popov 1979 & 104.3 & 0.98 & 0.94 & 0.92 & Ótimo & 0.32 \\
Jensen-Haise 1963 & 97.4 & 0.92 & 0.94 & 0.87 & Ótimo & 0.30 \\
Kimberly-Penman 1972 & 110.1 & 0.99 & 0.80 & 0.79 & Muito Bom & 0.71 \\
Benevides-Lopez 1970 & 96.6 & 0.82 & 0.74 & 0.60 & Sofrível & 0.48 \\
Kimberly-Penman 1982 & 99.1 & 0.71 & 0.82 & 0.58 & Sofrível & 0.61 \\
Priestley-Taylon 1972 & 84.2 & 0.85 & 0.59 & 0.50 & Mau & 1.13 \\
Penman modificado-FAO24 & 124.8 & 0.99 & 0.48 & 0.48 & Mau & 1.74 \\
Hargreaves-Samani 1985 & 81.7 & 0.89 & 0.49 & 0.43 & Mau & 1.31 \\
Makkink 1957 & 65.8 & 0.92 & 0.35 & 0.32 & Péssimo & 2.36 \\
Camargo 1971 & 81.9 & 0.70 & 0.51 & 0.35 & Péssimo & 1.32 \\
Tanque Classe A & 119.9 & 0.44 & 0.43 & 0.19 & Péssimo & 1.65 \\
Hamon 1961 & 60.7 & 0.53 & 0.28 & 0.15 & Péssimo & 2.74 \\
\hline
\end{tabular}

* r: coeficiente de correlação; d: índices de exatidão; c: coeficientes de desempenho; EPE: erro padrão de estimativa.

Tabela 3. Valores de coeficiente de correlação, índices de exatidão, coeficientes de desempenho e erro padrão de estimativa, referentes ao período úmido em Mossoró-RN, de 2002 a 2008.

\begin{tabular}{lcccccc}
\hline Equações & $\mathbf{\%}$ & $\mathbf{r}$ & $\mathbf{d}$ & $\mathbf{c}$ & Desempenho & EPE (mm.dia' \\
\hline Kimberly-Penman 1972 & 101,1 & 0,99 & 0,98 & 0,97 & Ótimo & 0,21 \\
Penman 1948 & 105,5 & 0,99 & 0,96 & 0,95 & Ótimo & 0,26 \\
Frere-Popov 1979 & 105,5 & 0,99 & 0,96 & 0,95 & Ótimo & 0,26 \\
FAO24-Radiação & 108,0 & 0,97 & 0,91 & 0,89 & Ótimo & 0,39 \\
FAO24-Blaney-Cliddle & 99,7 & 0,91 & 0,95 & 0,86 & Ótimo & 0,27 \\
Kimberly-Penman 1982 & 108,5 & 0,96 & 0,89 & 0,85 & Muito Bom & 0,55 \\
Penman modificado-FAO24 & 115,7 & 0,99 & 0,80 & 0,80 & Muito Bom & 0,76 \\
Hargreaves-Samani 1985 & 103,2 & 0,84 & 0,87 & 0,73 & Bom & 0,38 \\
Benevides-Lopez 1970 & 111,8 & 0,89 & 0,77 & 0,69 & Bom & 0,62 \\
Camargo 1971 & 107,3 & 0,77 & 0,83 & 0,63 & Mediano & 0,57 \\
Jensen-Haise 1963 & 116,9 & 0,89 & 0,71 & 0,63 & Mediano & 0,82 \\
Priestley-Taylon 1972 & 108,4 & 0,77 & 0,80 & 0,61 & Mediano & 0,56 \\
Tanque Classe A & 106,8 & 0,74 & 0,73 & 0,54 & Sofrível & 0,99 \\
Makkink 1957 & 80,5 & 0,85 & 0,57 & 0,49 & Mau & 0,96 \\
Hamon 1961 & 82,8 & 0,85 & 0,56 & 0,48 & Mau & 0,88 \\
\hline
\end{tabular}

* r: coeficiente de correlação; d: índices de exatidão; c: coeficientes de desempenho; EPE: erro padrão de estimativa. 
Os métodos que apresentaram resultados satisfatórios em ambos os períodos estudados foram Penman 1948, FAO24-Radiação, FAO24-BlaneyCriddle e Frere-Popov, classificados como ótimos de acordo com o coeficiente de desempenho. O método da FAO24-Blaney-Criddle apresenta grande praticidade, pois requer somente dados de temperatura do ar e algumas informações médias do clima, sendo adequado para estimativas mensais de ETo. Chiew et al. (1995) avaliaram o desempenho desse método em relação a Penman-Monteith e obtiveram coeficiente de correlação superior a 0,8 em 15 de 16 localidades estudadas na Austrália, para estimativas mensais de ETo.

Figura 2. Relação entre a evapotranspiração de referência estimada por diferentes métodos e o método Pemana-Monteith FAO 56, para o período úmido em Mossoró-RN, de 2002 a 2008.
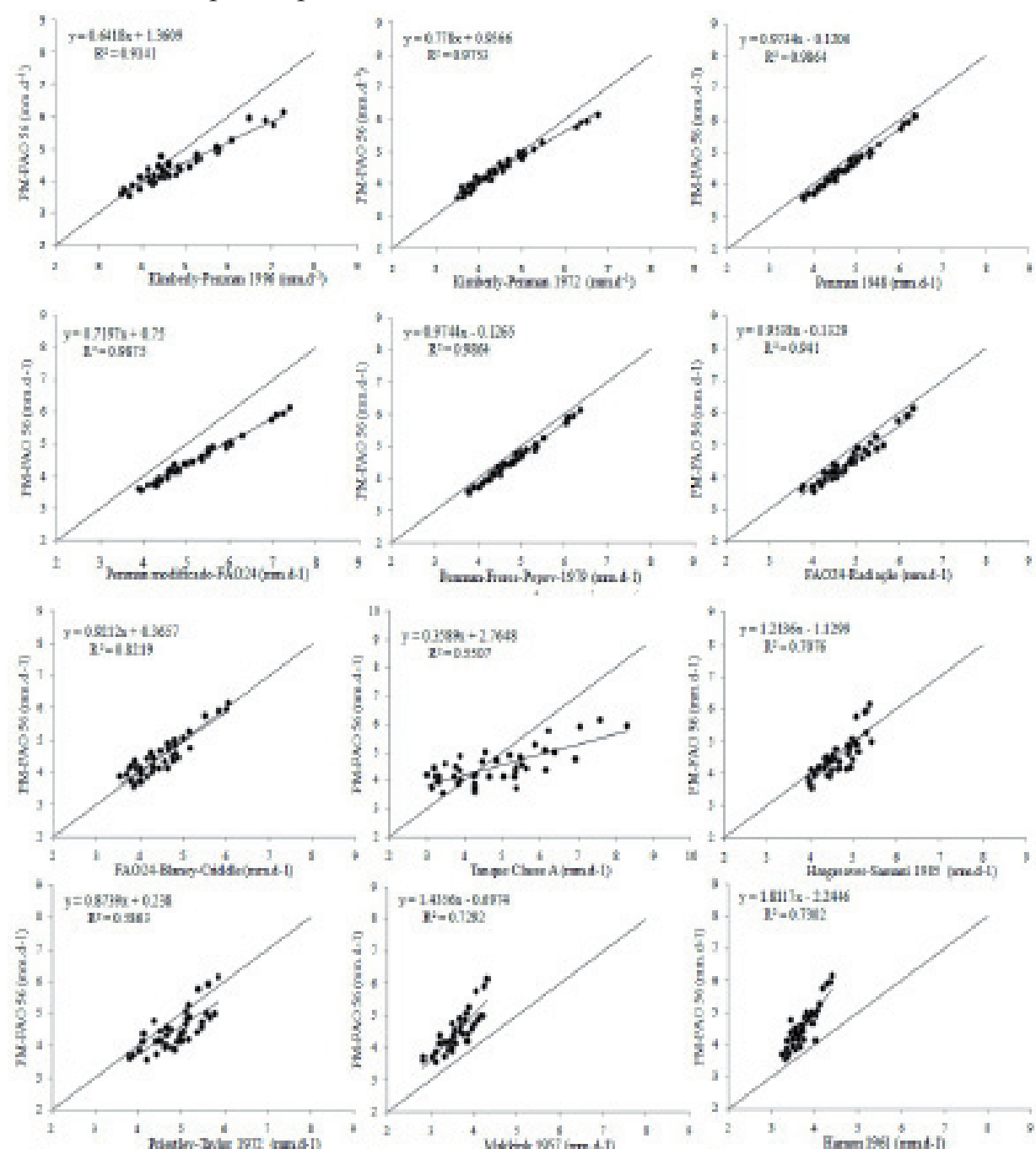

$$
\text { 3. } y=1 \mathrm{~min} k \mathrm{k}- \pm 2 \pi 6
$$
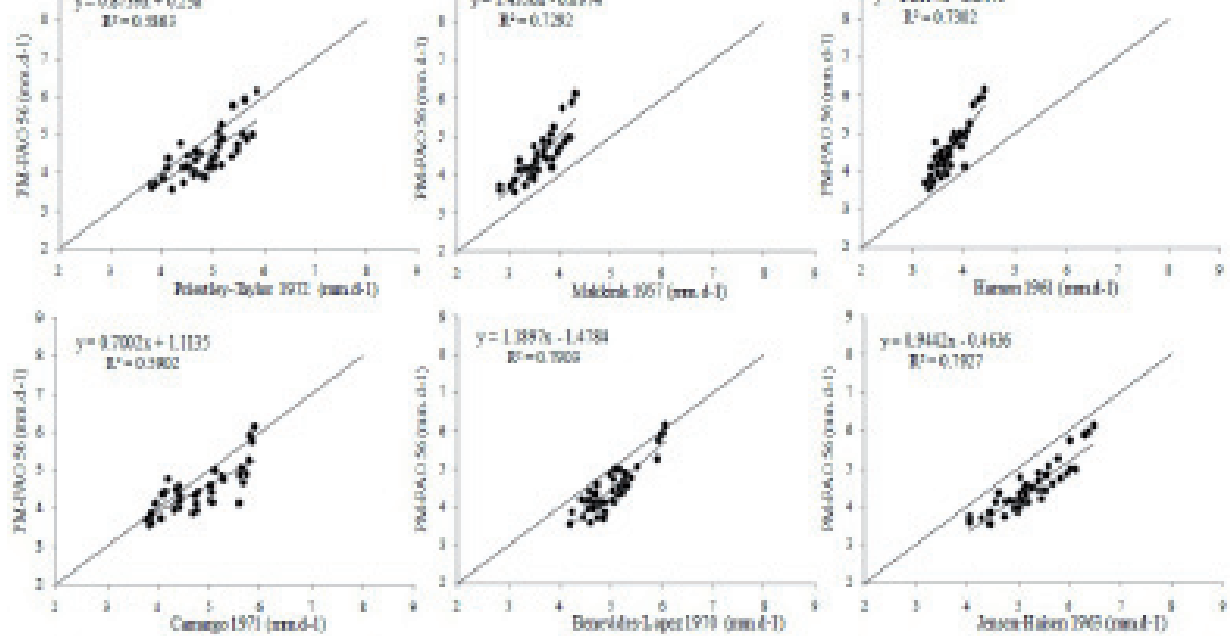
Figura 3. Relação entre a evapotranspiração de referência estimada por diferentes métodos e o método Pemana-Monteith FAO 56, para o período seco em Mossoró-RN, de 2002 a 2008.
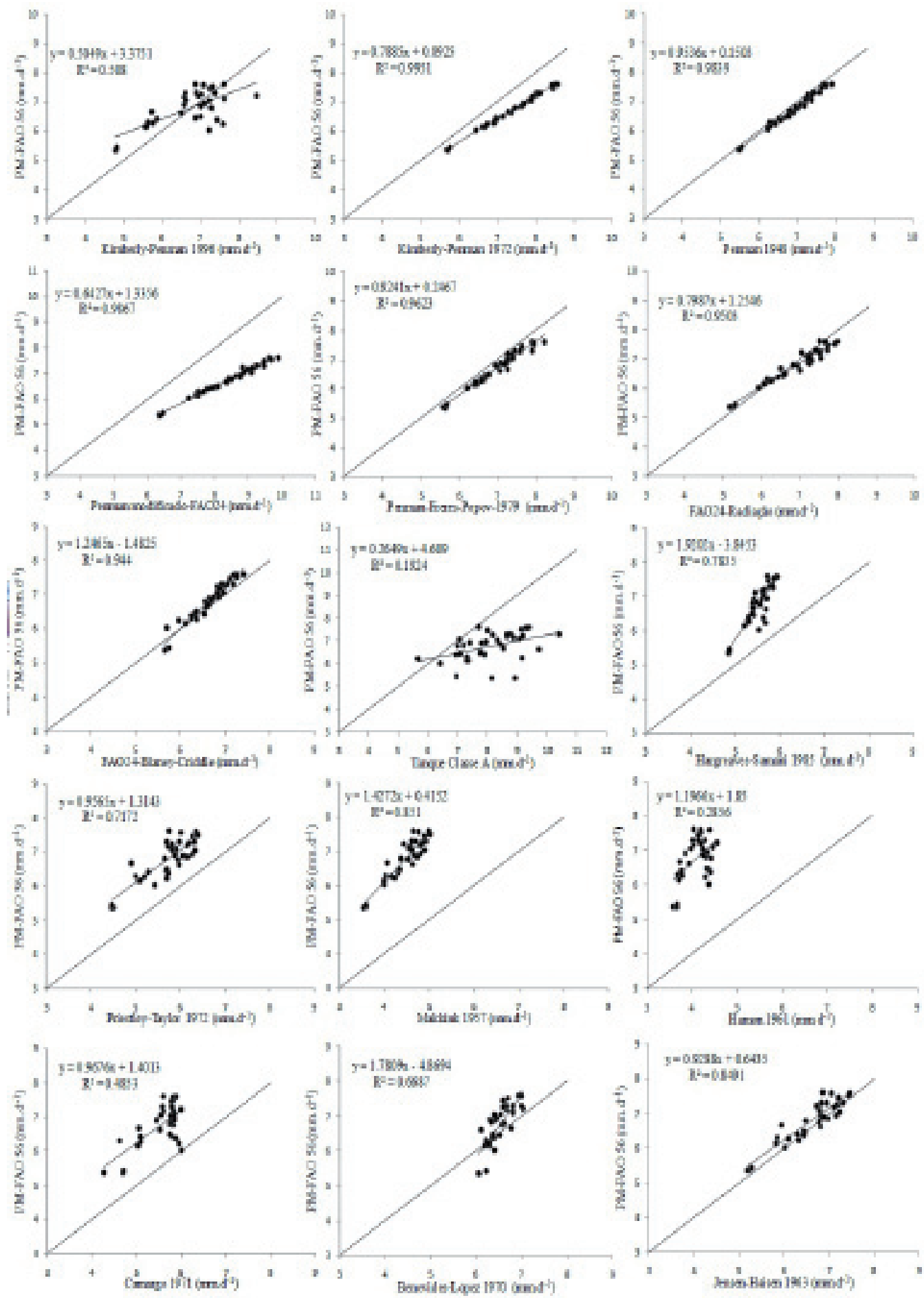

\section{Conclusões}

De todos os médodos estudados quatro deles (Penman 1948, FAO24-Radiação, FAO24-Blaney-
Criddle e Frere-Popov) apresentaram ótimo desempenho na estimativa da evapotranspiração de referência em qualquer época do ano, podendo serem recomendados para o uso nos projetos de 
irrigação na região, uma vez que seus resultados apresentaram uma satisfatória correlação com o método padrão, Penman-Montheith FAO 56.

De uma maneira geral os métodos testados mostraram uma melhora na precisão da estimativa, do período seco para o úmido, uma vez que nenhum deles foi considerado "péssimo" no primeiro semestre estudado.

\section{Referências}

ALLEN, R. G. REF-ET: reference evapotranspiration calculator, Version 2.1. Idaho: Idaho University, 2001. 82 p. (Software).

ALLEN, R. G.; PEREIRA, L. S.; RAES, D.; SMITH, M. Guidelines for computing crop water requeriments. Rome: FAO, 1998. 310 p. (Irrigation and Drainage Paper, $56)$.

BENEVIDES, J. G.; LOPEZ, D. Formula para el caculo de la evapotranspiracion potencial adaptada al tropico $\left(15^{\circ} \mathrm{N}-15^{\circ} \mathrm{S}\right)$. Agronomia Tropical, Maracay, v. 20, n. 5, p. 335-345, 1970.

BLANEY, H. F.; CRIDDLE, W. O. Determining water requirements in irrigated areas from climatological and irrigation data. Washington: USDA Soil Conservation Service, 1950. 48 p. (Technical Paper, n. 96).

BORGES,A.C.; MENDIONDO, E. M. Comparação entre equações empíricas para estimativa da evapotranspiração de referência na Bacia do Rio Jacupiranga. Revista Brasileira de Engenharia Agrícola e Amiental, Campina Grande, v. 11, n. 3, p. 293-300, 2007.

CAMARGO, A. P. Balanço hídrico no estado de São Paulo. 3. ed. Campinas: IAC, 1971. 24 p. (Boletim, n. 116).

CAMARGO, A. P.; SENTElHAS, P. C. Avaliação do desempenho de diferentes métodos de estimativa da evapotranspiração potencial no estado de São Paulo. Revista Brasileira de Agrometeorologia, Santa Maria, v. 5, n. 1. p. 89-97, 1997.

CARMO FILHO, F. do; ESPÍNOLA SOBRINHO, J.; MAIA NETO, J. M. Dados meteorológicos de Mossoró (janeiro de 1988 a dezembro de 1990). Mossoró: ESAM, 1991. v. 4, 470 p.

CHIEW, F. H. S.; KAMALADASA, N. N.; MALANO, H. M.; McMAHON, T. A. Penman-Monteith, FAO-24 reference crop evapotranspiration and class-A pan data in Australia. Agricultural Water Management, Amsterdan, v. 28, n. 1, p. 9-21, 1995.

FIETZ, C. R.; SILVA, F. C.; URCHEI, M. A. Estimativa da evapotranspiração de referência diária para a região de Dourados, MS. Revista Brasileira de Agrometeorologia, Santa Maria, v. 13, n. 2, p. 225-250, 2005.

FRERE, M.; POPOV, G. Agrometeorological crop monitoring and forecasting. Rome, Italy: FAO, 1979. 64 p. (Fao Plant Production and Protection Paper, 17).

HAMON, W. R. Estimating potential evapotranspiration. Journal of Hydraulics Division ASCE, Nova Iorque, v. 87, n. 3, p. 107-120, 1961.

HARGREAVES, G. L.; SAMANI, Z. A. Reference crop evapotranspiration from temperature. Basin. Journal of the Irrigation and Drainage Division-ASCE, New York, v. 111, n. 1, p. 113-124. 1985.

IZÍDIO, L. R. Determinação dos parâmetros " $a$ " $e$ " $b$ " da equação de Angstron, para estimativa da irradiação solar global em Mossoró. RN. 1994. Monografia (Graduação em Agronomia) - Escola Superior de Agronomia de Mossoró, Rio Grande do Norte.

JENSEN, M. E.; BURMAN, R. D.; ALLEN, R. G. Evapotranspiration and irrigation water requirements. New York: ASCE, 1990. 332 p.

JENSEN, M. E.; HAISE, H. R. Estimating evapotranspiration from solar radiation. Journal of the Irrigation and Drainage Division-ASCE, New York, v. 4, n. 1, p. 15-41, 1963.

MAKKINK, G. F. Testing the Penman formula by means of lysimeters. Journal of the Institution of Water Engineers, v. 11, n. 3, p. 277-288. 1957.

MEDEIROS, A. T. Estimativa da evapotranspiração de referência a partir da equação de Penman-Monteith, de medidas lisimétricas e de equações empíricas, em Piraipaba, CE. 2002. Tese (Doutorado em Irrigação e Drenagem) - Escola Superior de Agricultura Luiz de Queiroz. Universidade de São Paulo, São Paulo.

MEDEIROS, P. V. Análise da evapotranspiração de referência a partir de medidas lisimétricas e ajuste estatístico de nove equações empíricas- teóricas com base na equação de Penman-Monteith. 2008. Dissertação (Mestrado em Hidráulica e Saneamento) - Escola de Engenharia de São Carlos. Universidade de São Paulo, São Paulo.

MEDEIROS, S. L. Avaliação de métodos de estimativa as evapotranspiração de referência para a região mesoclimática de Santa Maria-RS. Revista Brasileira de Agrometeorologia, Santa Maria, v. 6, n. 1, p. 105-109, 1998.

OliveIRA, A. D. de. Comparação de métodos de 
estimativa da evapotranspiração de referência utilizando dados de uma estação meteorológica convencional $e$ automática. 2003. Tese (Doutorado em Agronomia) - Faculdade de Ciências Agrárias e Veterinária. Universidade de São Paulo, Jaboticabal.

OLIVEIRA, M. A. A.; CARVALHO, D. F. Estimativa da evapotranspiração de referência e da demanda suplementar de irrigação para o milho (Zea mays L.) em Seropédica e Campos, Estado do Rio de Janeiro. Revista Brasileira de Engenharia Agrícola e Ambiental. Campina Grande, PB, v. 2, n. 2, p. 132-135, 1998.

OLIVEIRA, R. Z.; OLIVEIRA, L. F. C.; WEHR, T. R.; BORGES, L. B.; BONOMO, R. Comparação de metodologias de estimativa da evapotranspiração de referência para a região de Goiânia, GO. Biosci. J., Uberlândia, v. 21, n. 3, p. 19-27, 2005.

PENMAN, H. L. Natural evaporation from open water, bare soil and grass. Proceedings of Royal Society-Series A, London, v. 193, p. 120-145, 1948.

PEREIRA, A. R.; VILLA NOVA, N. A.; SEDYIAMA, G. C. Evapo(transpi)ração. Piracicaba: FEALQ, 1997. $183 \mathrm{p}$.

PRIESTLEY, C. H. B.; TAYLOR, R. J. On the assessment of surface heat flux and evaporation using large-scale parameters. Monthly Weather Rev., Boston, v. 100, n. 2, p. 81-92, 1972.

SEDIYAMA, G. C. Evapotranspiração: necessidades de água para as plantas cultivada. Brasília, DF: ABEAS, 1998, 181 p. (Curso de especialização por tutoria à distância).

VESCOVE, H. V.; TURCO, J. P. Comparação de três métodos de estimativa da evapotranspiração de referência para a região de Araraquara-SP. Engenharia Agrícola, Jaboticabal, v. 25, n. 3, p. 713-721, 2005.

WRIGHT, J. L. New evapotranspiration crop coefficients. Journal of the Irrigation and Drainage Division-ASCE, New York, v. 108, n. 1, p. 57-74, 1982.

WRIGHT, J. L.; JENSEN, M. E. Peak water requerements of crops in Southern Idano. Journal of the Irrigation and Drainage Division-ASCE, New York, v. 96, n. 1, p. 193201, 1972. 